medgen $2012 \cdot 24: 84-85$

DOI 10.1007/s11825-012-0329-9

Online publiziert: 15. Juni 2012

๑) Springer-Verlag 2012
G. Gillessen-Kaesbach ${ }^{1} \cdot$ R. Siebert ${ }^{2}$

${ }^{1}$ Institut für Humangenetik, Universität zu Lübeck

${ }^{2}$ Institut für Humangenetik, Christian-Albrechts-Universität zu Kiel

\title{
Stellenwert der Array-basierten molekularen Karyotypisierung in der Humangenetik
}

ligation-dependant probe amplification“) wurde die Diagnostik von klinisch charakterisierten Mikrodeletions- und Mikroduplikationssyndromen gerade auch im Vergleich zum FISH-basierten Screening vereinfacht, blieb aber weiterhin auf die Subtelomerbereiche und bekannte Mikrodeletions- und duplikationsregionen beschränkt.

Aber erst durch die Einführung der molekularen Karyotypisierung in Form der initial auch als Matrix-CGH bezeichneten Array-CGH durch die Gruppen von Peter Lichter und Dan Pinkel [5] wurde die zytogenetische Diagnostik revolutioniert und die klassische Chromosomenanalyse in den Hintergrund gedrängt. Im Gegensatz zur klassischen Chromosomenanalyse können Array-basierte Verfahren der molekularen Karyotypisierung aber nur unbalancierte chromosomale Aberrationen detektieren, d. h. Zugewinn oder Verlust von genetischem Material. Single-nucleotide-polymorphism(SNP)-basierte Verfahren erlauben auch den Nachweis von Regionen mit kopienzahlneutralem Verlust der Heterozygotie bzw. von uniparentalen Isodisomien und Mosaiken [1]. Deshalb ergänzen sich beide Methoden derzeit mit unterschiedlichen Indikationen, wobei der Nachweis gerade balancierter Chromosomenveränderungen, z. B. in der Diagnostik von Fertilitätsstörungen oder von übertragbaren Chromosomenveränderungen, nach wie vor die Domäne der konventionellen Chromosomenanalyse ist. Balancierte Translokationen bei einem der Eltern stellen ein hohes Risiko für unbalancierte Chromosomenaberrationen beim zu erwartenden Kind dar. Hier wurden hoch- auflösende Oligonukleotidarrays entwickelt, die eine sichere Diagnostik z. B. im Rahmen der Präimplantationsdiagnostik (PID) ermöglichen.

Die Array-Technik wurde zunächst überwiegend in der Tumordiagnostik eingesetzt, wird aber heute sowohl zur Abklärung einer geistigen Behinderung [4], einer unklaren Entwicklungsstörung oder von Fehlbildungssyndromen benutzt. Die methodische Entwicklung der Array-Diagnostik ist mittlerweile soweit fortgeschritten ist, dass sie als Routinemethode in den meisten Laboren angewandt wird. Allerdings ist die klinische Interpretation der Befunde häufig schwierig. Viele der gefundenen Kopienzahlvarianten („copy numer variants“, CNVs) sind selten und ihre Bedeutung ist unklar. Auch die Bedeutung einer CNV bei einem gesunden Elternteil ist häufig schwer abzuschätzen. Die meisten dieser CNVs sind aber anscheinend nicht pathogen [6], sondern als Polymorphismen anzusehen, die mit einer Frequenz von $>1 \%$ in der Bevölkerung vorkommen. Unterschiedliche Arbeitsgruppen haben einen Algorithmus entwickelt, mit dem man die Pathogenität von CNVs einschätzen kann [3].

In diesem Themenheft wird auf die Bedeutung der Array-basierten molekularen Karyotypisierung im prä- und postnatalen Kontext sowie in der Tumorgenetik eingegangen.

Die wissenschaftliche Koordination des vorliegenden Themenschwerpunkts erfolgte unter Leitung von Prof. Dr. med. Gillessen-Kaesbach, Lübeck, und Prof. Dr. med. Reiner Siebert, Kiel. 


\section{Array-basierte Detektion chromosomaler Imbalancen im Kontext postnataler Diagnostik}

Hackmann et al. geben einen Überblick über die unterschiedlichen Technologien wie Oligonukleotidarray und SNPArray und diskutieren die Frage, ob ein $\mathrm{CNV}$ pathogen oder nicht pathogen ist. Außerdem wird auf gängigen Datenbanken (DGV, DECIPHER, ECARUCA und ISCA) eingegangen. Oneda und Rauch stellen in ihrem Beitrag die Bedeutung der Array-CGH-Diagnostik bei der Abklärung neurokognitiver Entwicklungsstörungen dar. In mehr als 18\% der Fälle kann mithilfe der molekularen Karyotypisierung eine ursächliche Chromosomenaberration festgestellt werden. Sie weisen darauf hin, dass Deletionen, insbesondere, wenn sie de novo auftreten, i. d. R. eine höhere Wahrscheinlichkeit aufweisen, krankheitsverursachend zu sein, als Duplikationen. Interessant ist die Beobachtung, dass chromosomale Mosaike mittels einer Array-CGH-Technik besser detektiert werden, als es die konventionelle Chromosomenanalyse vermag. Caliebe et al. berichten über die Auswertung der Daten von 1272 Patienten mit ungeklärter Entwicklungsverzögerung, die mithilfe der Array-CGH-Technik in Schleswig-Holstein untersucht wurden. In dieser Gruppe konnte etwa bei $10 \%$ eine relevante chromosomale Imbalance nachgewiesen werden. Insbesondere wird auch über rekurrente Veränderungen im Vergleich zur Literatur berichtet.

\section{Array-basierte Detektion chromosomaler Imbalancen im Kontext pränataler Diagnostik}

Held et al. gehen auf die Schwierigkeiten der Array-CGH-Technik in der Pränatalund Präimplantationsdiagnostik (PID) ein. Die Autoren sind der Meinung, dass aufgrund der nicht immer eindeutigen Bedeutung der Kopienzahlveränderungen (CNVs) eine Routineanwendung in der pränatalen Diagnostik nicht sinnvoll ist. Allerdings kann diese Methode sinnvoll sein, wenn man sie zur ursächlichen Abklärung von Auffälligkeiten im Ultraschall heranzieht. Auch eine weitere Abklärung von strukturellen Auffälligkeiten, die mittels einer konventionellen Chromosomenanalyse festgestellt wurde, wird von den Autoren als sinnvoll erachtet.

\section{Array-basierte Detektion chromosomaler Imbalancen im Kontext der Tumordiagnostik}

Baudis et al. geben eine Übersicht über die speziellen Aspekte der Array-basierten molekularen Karyotypisierung, die sich aus der somatischen Entstehung der chromosomalen Aberrationen in Tumoren ergeben. Sie diskutieren den Einsatz Arraybasierter Technologien, z. B. zur Charakterisierung pathogenetisch relevanter Imbalancen bei Tumoren, zur Definition von molekularen und klinischen Subgruppen und zur Identifizierung von Zielgenen für eine individualisierte Therapie. In einer Metaanalyse von über 22.000 Array-Analysen stellen die Autoren zudem die Muster der Aberrationen bei häufigen Tumoren sowie exemplarisch die unterschiedlichen Muster chromosomaler Imbalancen in histologischen Subgruppen von Tumoren dar.

Prof. Dr. Gabriele Gillessen-Kaesbach Prof. Dr. Reiner Siebert

\section{Korrespondenzadresse}

Prof. Dr. G. Gillessen-Kaesbach

Institut für Humangenetik

Universität zu Lübeck

Ratzeburger Allee 160, 23538 Lübeck

g.gillessen@uk-sh.de

Prof. Dr. R. Siebert

Institut für Humangenetik

Christian-Albrechts-Universität zu Kiel

Schwanenweg 24, 24105 Kiel

rsiebert@medgen.uni-kiel.de

\section{Literatur}

1. Conlin LK et al (2010) Mechanisms of mosaicism, chimerism and uniparental disomy identified by single nucleotide polymorphism array analysis. Hum Mol Genet 19(7):1263-1275

2. Flint J, Knight S (2003) The use of telomere probes to investigate submicroscopic rearrangements associated with mental retardation. Curr Opin Genet Dev 13:310-316

3. Gijsbers AC, Lew JY, Bosch CA et al (2009) A new diagnostic workflow for patients with mental retardation and/or multiple congenital abnormalities: test arrays first. Eur J Hum Genet 17:1394-1402
4. Hochstenbach R, Buizer-Voskamp JE, Vorstman JA, Ophoff RA (2011) Genome arrays for the detection of copy number variations in idiopathic mental retardation, idiopathic generalized epilepsy and neuropsychiatric disorders: lessons for diagnostic workflow and research. Cytogenet Genome Res 135:174-202

5. Pinkel D, Segraves R, Sudar D et al (1998) High resolution analysis of DNA copy number variation using comparative genomic hybridization to microarrays. Nat Genet 20:207-211

6. Vermeesch JR, Balikova I, Schrander-Stumpel C, Fryns JP, Devriendt K (2011) The causality of de novo copy number variants is overestimated. Eur J Hum Genet 19:1112-1113 